\title{
Research on Adjustment of Preliminaries Caused by New Work Sections And Trades added in list
}

\author{
LI Yanqiu ${ }^{1, a}$ and KE Hong ${ }^{2}$ \\ ${ }^{1,2}$ School of Management, Tianjin University of Technology, Tianjin, China \\ a748066496@qq.com
}

Keywords: Bill of quantities; Preliminaries; Adjustment of cost

\begin{abstract}
The new work sections and trades added in list will cause adjustment of preliminaries. For both parties concerned, the determination of preliminaries cost adjustment is the focus points of controversy. The study about the research on adjustment of preliminaries is rare. Therefore, through literature research and combination with the provision for preliminaries cost adjustment based on code of bills of quantities and valuation for construction works(13 list), this paper defines the scope of new work of sections and trades added in list, analyzes the impact of new work of sections and trades added to preliminaries cost adjustment. This paper discusses how to adjust the preliminaries cost from the increasing of amount of preliminaries and missing items of preliminaries.
\end{abstract}

\section{Introduction}

At present, there are some problems in our country's engineering construction practice, which is due to the new work sections and trades added in list, the disputes of contract price adjustment are also increasing. Those have become a serious problem in the Construction practice. The new work sections and trades added in list will not only increase the amount of original preliminaries, but also lead to the item of preliminaries adding or missing. It has become a focus and hot issues in the engineering construction that how to reasonably define the scope of the preliminaries and explicit the missing items of preliminaries and the increasing of amount of preliminaries which caused by the new work sections and trades added in list. Therefore, it is necessary to make a research on adjustment of preliminaries, which is to protect the interests of both parties.

In the field of project management research, the research on the preliminaries is mainly reflected in the adjustment of total lump sum preliminaries[1,2], the item of preliminaries lack ${ }^{[3,4]}$, discrepancies in item description $^{[5]}$ and so on. Yan Ling et proposed three kinds of adjustment conditions and methods about preliminaries caused by engineering quantity changes ${ }^{[6]}$. Wu Guoyou discussed how to calculate preliminaries fee and adjust or determine preliminaries fee in the process of final account settlement from the characteristics and contents of bill of quantities (BQ) and the calculation of preliminaries in engineering practice ${ }^{[7]}$. Li Han made a deeply research on how to reduce the change of preliminaries fee through analyzed the role of the preliminaries in the engineering practice ${ }^{[8]}$. Obviously, the relevant scholars are mainly focus on the lack of preliminaries and discrepancies in item description when study the adjustment of preliminaries. The study of the adjustment of preliminaries caused by new work sections and trades added in list is very few. Therefore, this paper discuss the problem of preliminaries adjustment caused by new work sections and trades added in list according to the contract price adjustment provisions from the 13 list. Thus it can solve the focus problem of both contract parties.

Define the scope of preliminaries adjustment caused by new work sections and trades added in list

\section{Define the scope of new work sections and trades added in list}

(1) Tenderer misses the item in the preparation of BQ for tendering. When the depth of the design drawings is not enough or the depth of design did not meet the requirements of the construction organization, tenderer will miss the item list easily in the preparation of BQ for tendering. If the tenderer have less practical construction experience, or not familiar with the 
construction technology, construction process and construction specification, it will lead to item list missing easily in the preparation of BQ. There are no such items in the contract which can be regarded as the employer has included in its corresponding entity in the project.

(2) Variation order caused by the change of construction scheme for non contractor's reason. Because of the construction conditions and environment do not match the original design drawings or related data information, It will always appear item added in the process of construction. This will need draw up a new construction scheme based on the original construction plan, and the corresponding preliminaries also need make a change.

\section{The impact of new work sections and trades added in list to preliminaries}

It is necessary to make the impact of new work sections and trades added in list to preliminaries clearly before the research on the path of the preliminaries adjustment. From the content and composition of preliminaries, according to the calculation of the unit price of preliminaries is mainly related to its transport, setting, demolish and storage. But the sun lump calculation of preliminaries is mainly related to the protection in the special environment or other factors. The adjustment of preliminaries caused by new work sections and trades added in list can be divided into three categories: preliminaries related to sections and trades or duration, and related to construction organization design scheme, and the relevant preliminaries about rate and engineering materials.

The change of preliminaries caused by new work sections and trades added in list can be adjusted from the "quantity" and "item" two paths. In view of the above analysis, we can get the whole influence process of new work sections and trades added in list to preliminaries adjustment. The specific adjustment path as shown in figure 1 below:

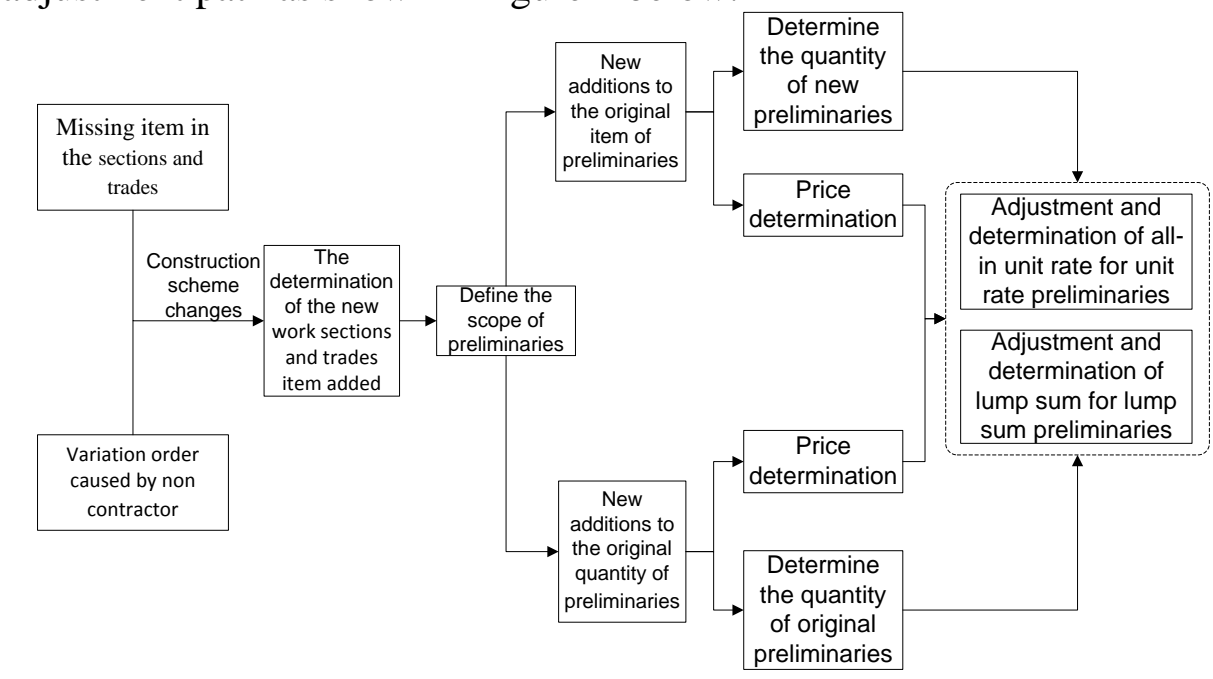

Figure 1 Adjustment path of preliminaries

As shown by Figure 1, the new work sections and trades added in list may cause new additions to the original item of preliminaries, or may cause new additions to the original quantity of preliminaries. The former can be regard as the addition of preliminaries' item, and the later can be regard as the addition of preliminaries' quantity. In this paper, we will make a detailed analysis on how to adjust the preliminaries in these two aspects.

The price adjustment of preliminaries' quantity added caused by the new work sections and trades added in list

\section{The method of price adjustment about unit rate preliminaries' quantity added}

There are clear rules for the adjustment of the preliminaries' quantity added caused by the new work sections and trades added in the 13 list. These provisions provide theoretical basis for the study of contract price adjustment caused by preliminaries' quantity added. Now, summary the provisions of preliminaries price adjustment which calculated in unit rate as follows: 
Table1 Analysis on the contract price adjustment caused by unit rate preliminaries' quantity added in 13 list

\begin{tabular}{|c|c|c|c|}
\hline Clause No. & $\begin{array}{c}\text { the direction of } \\
\text { adjustment }\end{array}$ & \multicolumn{1}{c|}{ The contract price adjustment provisions } \\
\hline 9.5 .2 & $\begin{array}{c}\text { After adding a new work sections and trades that caused the change of } \\
\text { the reason of } \\
\text { adjustment }\end{array}$ & $\begin{array}{l}\text { preliminaries, it shall be in accordance with the provision of 9.3.2 code. The } \\
\text { contract price shall be adjusted contract price after the contractor to submit } \\
\text { the plan approved by the employer. }\end{array}$ \\
\hline 9.3 .1 & $\begin{array}{l}\text { the way of } \\
\text { adjustment }\end{array}$ & $\begin{array}{l}\text { If there are applicable item to the change in the BQ, They should adopt the } \\
\text { changes caused variation order, and the deviation is more than 15\%, the unit } \\
\text { rate preliminaries shall be in accordance with the provision of 9.3.2 code. }\end{array}$ \\
\hline 9.3 .2 & $\begin{array}{l}\text { When the change of engineering causes the construction plan and } \\
\text { preliminaries item to change and the contractor submit adjustment of } \\
\text { preliminaries fee, they should submit the scheme to be implemented to } \\
\text { employer for confirming and should be explained in detail and compared with } \\
\text { the original plan. The proposed scheme can be implemented through } \\
\text { confirmation by both parties, } \\
\text { and adjust preliminaries in accordance with the following provisions. The } \\
\text { preliminaries to be used in the calculation of the unit rate price shall be } \\
\text { adjustment } \\
\text { unit rate } \\
\text { calculation }\end{array}$ \\
\hline
\end{tabular}

In the study of original preliminaries' quantity changes, the adjustment provisions of engineering quantity change range is that when the amount of project is added more than $15 \%$, the all-in unit rate of increased part should be reduced; when the amount of project is reduced more than $15 \%$, the all-in unit rate of remaining should be raised. If the increase of the original preliminaries' quantity have applicable item, its scope of application is that the lack of preliminaries is similar to existing projects in the construction conditions, technology, methods, drawings on the size and material, and the construction time is not on the critical line.

The method of price adjustment about the increasing of cardinal number about lump sum preliminaries

The increasing of engineering quantity for lump sum preliminaries is not reflected in the amount of preliminaries, It is determined by the combination of artificial, material and mechanical caused by variation order and their rates. Therefore, when the sections and trades added, the increase of cardinal number will make the largest impact to the adjustment of lump sum preliminaries.

According to 13 list, the preliminaries about lump sum calculation should be adjusted according to the actual change, but it should consider the floating factor of contractor's. That is to say the final adjustment price is calculated in accordance with the actual price multiplied by floating rate of contractor. For the cardinal number of lump sum preliminaries, each region is different. But 13 list is just have the fixed labor cost or fixed labor plus fixed machinery cost as calculation base. In the actual project, the calculation base of additional cost for night work is labor plus machinery cost, work sections and trades cost, and engineering fees. The calculation base of additional cost for winter rainy season is labor cost and labor plus machinery cost, work sections and trades cost, and engineering fees and so on.

\section{The price adjustment of preliminaries missing caused by the new work sections and trades added in list}

\section{The method of price adjustment about unit rate preliminaries item missing}

There are clear rules for the adjustment of the preliminaries item missing caused by the new work sections and trades added in the 13 list. These provisions provide theoretical basis for the study of contract price adjustment caused by preliminaries item missing. Now, summary the provisions of preliminaries price adjustment which calculated in unit rate as follows: 
Table2 Analysis on the contract price adjustment caused by unit rate preliminaries missing in 13 list

\begin{tabular}{|c|c|c|}
\hline $\begin{array}{l}\text { Clause } \\
\text { No. }\end{array}$ & $\begin{array}{c}\text { the direction of } \\
\text { adjustment }\end{array}$ & The contract price adjustment provisions \\
\hline 9.5 .2 & $\begin{array}{l}\text { the reason of } \\
\text { adjustment }\end{array}$ & $\begin{array}{l}\text { After adding a new work sections and trades that caused the change of } \\
\text { preliminaries, it shall be in accordance with the provision of } 9.3 .2 \text { code. The } \\
\text { contract price shall be adjusted contract price after the contractor to submit the } \\
\text { plan approved by the employer. }\end{array}$ \\
\hline 9.3 .1 & $\begin{array}{l}\text { the way of } \\
\text { adjustment }\end{array}$ & $\begin{array}{l}\text { 1. If there are applicable item to the change in the BQ, They should adopt the } \\
\text { unit price of the project. } \\
\text { 2. If there are no applicable item but have similar item to the change in the BQ, } \\
\text { They should adopt the unit price of the project. They can refer to the unit rate } \\
\text { price in a reasonable range. } \\
\text { 3. If there are no applicable or similar item to the change in the BQ, They use } \\
\text { information price and consider the floating factor for contractor quotation. } \\
\text { 4.If there are no applicable item, similar item information price to the change in } \\
\text { the BQ, They can use market price. }\end{array}$ \\
\hline 9.3 .2 & $\begin{array}{l}\text { preliminaries } \\
\text { adjustment for } \\
\text { unit rate } \\
\text { calculation }\end{array}$ & $\begin{array}{l}\text { The change of construction scheme caused by variation order makes } \\
\text { preliminaries changed should used unit rate price to calculate, These should be } \\
\text { in accordance with the actual changes in the provision of } 9.3 .2 \text { code. }\end{array}$ \\
\hline
\end{tabular}

From table 2 we can see 13 list have done a division from its source and direction to the adjustment of unit rate preliminaries caused by the new work sections and trades added in list. This section is mainly to study the new path of the preliminaries adjustment in the sections and trades, that is to say there are no preliminaries in the original list, the price adjustment of preliminaries are divided into no applicable item but have similar item and no applicable and similar item but have information price or market price.

The price adjustment methods of preliminaries missing about calculating on the lump sum

Preliminaries for calculated on the lump sum can be divided into competitive and non competitive projects. In the contract, the cost of lump sum project always uses the lump sum to calculate and adjust the preliminaries according to what actually happened. But we should consider the floating factor for contractor quotation. That is, the final price is calculated by the actual adjustment price multiplied by the floating factor. For the cost change caused by the quantity of sections and trades change, the preliminaries fee will be changed. When lump sum preliminaries changed caused by engineering leakage, the Contractor shall be entitled to put forward adjusting preliminaries fee. The cost of lump sum preliminaries caused by engineering leakage or variation order should be calculated by contractor according to the actual preliminaries and the rate which based on original calculation, and consider the floating factor rate.

\section{Conclusion}

The analysis and Research of the preliminaries adjustment caused by the new work sections and trades added in list need to be based on code of bills of quantities and valuation for construction works (GB50500-2013), this paper defined the scope of the situation of new work sections and trades added in list, and analyzed the influence of new work sections and trades added in list to preliminaries. Two different methods of calculation are putted forward in this article, that is unit rate Calculation method and lump sum Calculation method, which is based on the adjustment of new additions to the original item and quantity of preliminaries. In the present engineering practice, the research on the adjustment of preliminaries fee caused by the new work sections and trades added in list provide a good basis for both contract parties, it improves the ability of the contractor to adjust the preliminaries fee in the engineering practice, and provides a solid foundation for the healthy development of engineering practice. 


\section{References}

[1] L. Yan, L. Chen, J. Hu. Adjustment of Total Lump Sum Preliminaries under Code of Bills of Quantities and Valuation for Construction Works. Journal of Engineering Management. 2013, 27(6):92-96.

[2] H.X. He, X.G., L. Chen. Research on the adjusting the total measure project price under the mode of BOQ pricing 2013rd edition. Engineering economy. 2014(9):9-15.

[3] L. Yan, J.P. Li, J. Hu. Study on the Responsibility and Price Adjustment Condition of the Measures in the Bill of Quantities in the Bill of Quantities. Building economy, 2013(11):45-47.

[4] C. Wang. Study on the Price Adjustment Caused by the Missing Items of the Preliminaries in Bill of Quantities. Value engineering, 2014(35):81-82.

[5] D.Y. He, H.R. Tang, H. Yin. Research on Cost of Preliminaries Adjustment for Item Characteristic Discrepancy. Construction supervision and inspection and cost, 2014,7(4):5-8.

[6] Research on the Adjustment of Project Cost Based on the Change of Engineering Quantity. Building economy,, 2013 (9):46-50.

[7] G.Y. Wu. On the Reasonable Drawing of Measures in The Bill of Quantities Bill of Quantities. Building economy, 2011 (8):68-70.

[8] H. Lin. Project cost management for engineering quantity list. Journal of Huazhong University of Science and Technology, 2008,25(3):264-266. 